\title{
A Single-Lumen Central Venous Catheter for Continuous and Direct Intra-abdominal
}

\section{Pressure Measurement}

Oscar J.F. van Waes ${ }^{1}$, Jean B. Jaquet ${ }^{2}$, Wim C.J. Hop ${ }^{3}$, Marjolein J.M. Morak ${ }^{1}$, Jan M. Ijzermans ${ }^{1}$, Jan Koning ${ }^{4}$

${ }^{1}$ Department of Surgery, Erasmus University Medical Center Rotterdam, Rotterdam, The Netherlands,

${ }^{2}$ Department of Plastic Surgery, Medisch Centrum Rotterdam Zuid, Rotterdam, The Netherlands,

${ }^{3}$ Department of Epidemiology and Biostatistics, Erasmus University Rotterdam, Rotterdam, The Netherlands,

${ }^{4}$ Department of Surgery, Reinier the Graaf Hospital, Delft, The Netherlands.

\section{Address for Correspondence}

Oscar J.F. van Waes, MD

Department of Surgery/Traumatology

Erasmus University Medical Center

PO Box 2040

3000 CA, Rotterdam

The Netherlands

e-mail: ovanwaes@gmail.com 


\section{Abstract}

Background: Abdominal compartment syndrome (ACS) is associated with high morbidity and mortality rates. Therefore, the need for a good diagnostic tool to predict intra-abdominal hypertension (IAH) and progression to ACS is paramount. Bladder pressure (BP) has been used for several years for intra-abdominal pressure (IAP) measurement but has the disadvantage that it is not a continuous measurement. In this study, a single-lumen central venous catheter (CVC) is placed through the abdominal wall into the abdominal cavity to continuously and directly monitor the intraabdominal pressure (CDIAP). The aim of this study was to evaluate the use of CDIAP to measure BP as a representative of the true IAP.

Methods: Both BP and CDIAP were prospectively recorded on a variety of surgical patients admitted to the intensive care unit (ICU) from March 2003 up to December 2004. At the end of the surgical procedure, the CVC was placed through the abdominal wall and connected to a pressure transducer. In addition, the BP was measured through the urine drainage port after clamping the catheter and filling the bladder with $50 \mathrm{ml}$ of $0.9 \%$ saline. At least three paired measurements (BP and CDIAP) were performed for at least one day on the ICU in a standardized manner at preset time intervals on each patient. The paired measurements were compared using the Bland-Altman (B-A) method. Data are presented as mean \pm standard deviation.

Results: Over a period of 22 months (March 2003 until December 2004), 125 paired measurements of both BP and CDIAP were recorded on 25 patients. The mean age was $72.4 \pm$ 6.6 years. Eighteen patients underwent central vascular surgery, and seven patients with peritonitis received laparotomy. The mean CDIAP was $11.4 \pm 4.8$ (range 2-30) $\mathrm{mmHg}$, and the BP was $12.9 \pm 5.3$ (range 3-37) $\mathrm{mmHg}$. The mean difference between CDIAP and BP was $1.6 \pm 2.7 \mathrm{mmHg}$. There was an acceptable level of agreement (intraclass correlation 0.82) between IAP measured by BP and IAP measured via CDIAP. 
Conclusion: Continuous direct intra-abdominal pressure measurement proved that the BP measurement approach of Kron is representative of the IAP. CDIAP measurement is accurate and makes it easier for the nursing staff to be informed of the IAP.

Key Words Continuous intra-abdominal pressure measurement 


\section{Background}

Abdominal compartment syndrome (ACS) is associated with a high rate of morbidity and mortality [1-5]. Analogously to compartment syndrome of the extremities, ACS is a dynamic process that can develop rapidly over a period of just several hours [1]. If clinical signs occur, like cardiorespiratory failure and anuria, irreversible damage due to ischemia has already been done [2, 3, 6-8]. A good diagnostic tool for measuring the progression from intra-abdominal hypertension (IAH) to ACS, as defined by the World Society of ACS (IAH = IAP > 12 $\mathrm{mmHg}$ ) and ACS (IAP > $20 \mathrm{mmHg}+$ organ failure), is necessary. Various IAP measurement approaches have been used [9]. IAP measurement through the draining port of a urinary catheter, as described by Kron [10] and modified by Iberti [11] and Sugrue [12], has been chosen as the golden standard for IAP measurement during a consensus meeting at the World Congress for ACS in December 2004, Noosa, Australia. Though the bladder pressure (BP) is an accurate representative of the IAP, it is labor intensive because it is intermittent and so a rapid increase of IAP could be missed. A continuous pressure measurement device is therefore preferable. A promising alternative for continuous BP measurement has recently been presented by Balogh \& Sugrue [13]. Since the BP is only a representative of the real IAP, it could be biased by factors like the temperature of the liquid used as the water column or the muscle tone of the bladder; such potential biases have not been studied. In this study, we present a new and simple method for IAP measurement which is advantageous in that it is continuous and direct. 


\section{Methods}

The validation of continuous direct intra-abdominal pressure (CDIAP) versus BP was done prospectively over period of 22 months (March 2003 until December 2004) on patients recovering from surgery in the intensive care unit (ICU) of the Reinier de Graaf Hospital in Delft, a teaching hospital in The Netherlands. Prior to surgery, a standard silicone Foley catheter $\left(\right.$ BardBiocath $\left.^{\circledR}\right)$, size 18-Fr, was placed into the bladders of the patients enrolled in this study. At the end of the surgical procedure, a single-lumen central venous catheter (CVC) (ArrowBlue Flextip ${ }^{\circledR}$ ) was introduced into the abdomen perpendicular to the surface at approximately $5 \mathrm{~cm}$ cranial of the iliac crest in the anterior axillary line and fixated with a $3 / 0$ ethilon $\left(\right.$ Ethicon $\left.^{\circledR}\right)$. Continuous normal saline perfusion $(4 \mathrm{ml} / \mathrm{h})$ was maintained in the catheter, and it was connected to a pressure transducer (Medisize ${ }^{\circledR} 2006$ 2B) placed at the anterior thigh level with the pubic bone and then connected to the monitor (Figure 1). After the transducer was zeroed, the CDIAP could be read continuously. CDIAP was compared to the IAP as determined by the Kron technique [10]. In this technique, the drainage port of the Foley catheter was clamped after $50 \mathrm{ml}$ of sterile saline was injected into the bladder. The BP was then measured using a 16- gauge needle placed in the culture aspiration port the connect to the pressure transducer placed on the anterior thigh, level with the pubic bone, of the other leg and then connected to the pressure transducer and monitor (Figure 2). These paired measurements were done at four-hour intervals at least four times in each patient in the supine position. The degree of agreement between the two types of IAP measurement was evaluated using the Bland and Altman method [14, 15]. Mixed-model ANOVA, which takes into account inter-individual differences, was used to calculate the $95 \%$ confidence interval for the difference of means. Besides the paired measurements of CDIAP and BP, the type of surgical intervention and possible complications with the catheter were also recorded prospectively. The study was approved by the Human Research and Ethics Committee of the hospital and conducted with the informed consent of each participating patient. 


\section{Results}

Over a period of 22 months (March 2003 until December 2004), 125-paired measurements of both $\mathrm{BP}$ and CDIAP were recorded for 25 patients. The mean age $( \pm \mathrm{SD})$ was $72.4 \pm 6.6$ years. Eight out of the 25 patients enrolled were female. All patients underwent laparotomy; 15 patients had vascular surgery for aneurysm of abdominal aorta, of which six were ruptured, seven patients had peritonitis of various origins (perforation, dehiscence of anastomosis, etc.), and three patients had vascular surgery for occlusions of iliac arteries. The mean CDIAP was $11.4 \pm 4.8$ (range $2-30) \mathrm{mmHg}$, and the $\mathrm{BP}$ was $12.9 \pm 5.3$ (range 3-37) $\mathrm{mmHg}$. The mean difference (CDIAP minus BP) was $-1.6 \mathrm{mmHg}$, and the SD of the differences was $2.7 \mathrm{mmHg}$. The mean difference was significant $(p<0.001$, and the 95\% confidence interval for the mean difference ranged from -2.3 to $-0.9 \mathrm{mmHg}$ ). Figure 3 shows the Bland-Altman plot. This plot shows that the variation of the differences does not depend on the level of measurements. The difference in the means was $1.6 \mathrm{mmHg}$, which is $<2 \mathrm{mmHg}$ - accepted as the maximum mean difference during a consensus meeting at the World Congress for ACS in December 2004, Noosa, Australia. Although the difference can be large for individual measurements, the overall agreement is acceptable (intraclass correlation: 0.82; Figure 4). Only minor complications occurred when CDIAP was used: in one patient there was some leakage of ascites along the CVC, but this did not hinder CDIAP measurement and was not accompanied by infection. In another patient, the catheter was blocked with a blood cloth which could not be flushed, and it was removed after six readings. 


\section{Discussion}

Abdominal compartment syndrome is a syndrome which is no longer regarded as hype, but as a multiplex complication seen with a wide variety of surgical patients. This is highlighted by the exponential increase in the number of publications on this topic over the last few years [16]. Since ACS is increasingly being recognized as a complicating event during treatment of the seriously sick patient, it is important to understand the pathophysiology in order to prevent the development of IAH to ACS. Abdominal compartment syndrome is a dynamic process that develops according to the physical laws of Laplace, which state that pressure within a cylinder is directly proportional to its wall tension and inversely proportional to its radius. This implies that if the abdominal wall is stretched to its limits and the abdominal volume cannot expand any more, the IAP will incline rapidly and the patient will develop ACS over a short period of time. In another study, we demonstrated that ACS developed over a period of between 4 and $10 \mathrm{~h}$. Therefore, we conclude, in accordance with Balogh et al. [12, 13, 17], that there is a need for continuous IAP measurement, so rapid inclines of IAP will not be missed and interventions can be performed. In this study, both BP and CDIAP measurement techniques accurately detected an increase in IAP. Though an inclining trend in the IAP was noticed more swiftly by CDIAP compared to the BP measurements performed at intervals, this did not result in an alteration of the treatment strategy for the individual patients. Various approaches to continuous IAP measurement have been suggested [9], including IAP measurement using nasogastric tubes and tonometers $[8,18]$. There seems to be a difference between the values of IAP read by the nasogastric tubes and BP [19]. Obeid et al. [7] compared gastric, rectal and vesical pressure to IAP measured by means of the laparoscopic insufflator. He concluded that IAP measured by BP was the most reliable method. Other disadvantages of gastric pressure measurement using a water column as a medium for pressure measurement are that the pressure tends to fluctuate during migrating motor 
complexes [20, 21], it cannot be used during enteral feeding by a nasogastric tube, and it is relatively costly. Gastric pressure measurement by a catheter with an inflated balloon at the tip, as suggested by Spiegelberg, does not have the disadvantages mentioned above, but it does have the advantages of continuity and simplicity. Though it seems very promising, it needs further validation in the clinical setting. The method of continuously measuring BP as a representative of IAP, as proposed by Balogh et al. [13], appears promising but also has some issues that need clarification. It is not known whether the temperature of the infused saline, the position of the patient and the consciousness of the patient has any effect on the detrusor activity of the bladder wall, and therefore whether it could interfere with the BP readings.

Since all other methods of IAP measurement record the IAP in an indirect manner (i.e., not the true IAP value), we tested a simple set-up for measuring the IAP directly and continuously. Direct IAP measurements have been done in the laboratory setting in animal studies [10, 11, 22, 23] and laparoscopy [24, 25], but not on patients at risk of ACS in the ICU setting. Thus far, only one other study group (Shafik et al. [26]) has performed direct IAP measurements on a variety of patients - none of them in intensive care - by placing a Verres needle blindly into the abdomen. Though the author did not mention any complications, we think that this procedure is too hazardous and therefore should not be used. In comparison, in our approach, at the end of the operation the catheter is placed through the abdominal wall, under direct vision, similar to the way the subclavian vein is encountered, and the risk of puncturing intra-abdominal organs is minimal. Therefore, it is less invasive and hardly any hindrance to the patient who already is being operated upon and sedated.

We experienced no complications such as infections due to the catheter, and this method could be used during the total stay of the patient in the ICU without complications (up to ten days in this study). The only complications we observed were leakage along the catheter, but this did not interfere with the readings, and in another patient the catheter was blocked with a blood cloth that could not be flushed and had to be removed. All of our readings were done 
with the patient in the supine position, and there was no marked difference in IAP readings when the patient was unconscious or awake. Since the pressure was read continuously, it also has the benefit that is simple to monitor the abdominal perfusion pressure, APP (APP = mean arterial pressure minus IAP), which might be a better indicator of whether or not the patient is suffering from ACS [13, 27]. Other benefits of measuring CDIAP by means of CVC are that it requires no effort for the ICU nurses, who can easily monitor the IAP instead of the need to perform laborintensive BP measurements in the approach of Kron or Iberti [10, 11], and that it circumvents the additional risks of needle stick injury or urinary tract infection via the culture aspiration port. In addition, CDIAP measurement also appears cost effective in comparison to the $\mathrm{BP}$ technique. Whereas the $\mathrm{BP}$ measurement system is usually replaced every ten measurements because the saline bag is empty, the CDIAP measurement system is left in place during the entire period of admission into the ICU. The cost of one BP set, according to Kron, is approximately $€ 35$, with the main costs being the pressure system and the Foley catheter. The cost of the CDIAP measurement set is approximately $€ 50$, including a similar pressure system and a single-lumen CVC. If, for example, one was to follow a patient for six days (i.e., 36 BP measurements), it would require one CDAIP set-up (€50) versus four Kron BP systems $(4 \times 35=€ 140)$, resulting in a cost reduction of $€ 90$.

Our results revealed a high degree of correlation between BP and CDIAP. B-A statistics depicted a good level of agreement (mean difference $<2 \mathrm{mmHg}$ ), but there were some readings in which there was a fair discrepancy between BP and CDIAP. Since the CDIAP did not fluctuate in its values, this may be explained by inaccurate readings taken during BP measurement using the Kron technique, since this is a rather complex procedure. Continuous BP readings would circumvent this problem. The authors are therefore evaluating the correlation and agreement between CDAIP and continuous BP in another study. In conclusion, the authors advocate that patients at risk of developing ACS and who are also receiving laparotomy should be fitted with a CVC for CDIAP measurements, as described 
above. Patients who have not undergone laparotomy but are at risk of ACS (e.g., trauma, peritonitis) would best be monitored with the continuous BP technique, as suggested by Balogh et al. [13], but this should be validated in a prospective study comparing it to the CDIAP measured by CVC approach.

\section{Acknowledgments}

This study was performed independently without financial support. The authors wish to thank the intensivists and the nursing staff of the ICU of the Reinier de Graaf Hospital for their interest and support. 


\section{References}

1. Balogh Z, McKinley BA, Holcomb JB, Miller CC, Cocanour CS, Kozar RA, Valdivia A, Ware DN, Moore FA. Both primary and secondary abdominal compartment syndrome can be predicted early and are harbingers of multiple organ failure. J Trauma 2003;54:848-59, (discussion 859-61).

2. Moore AF, Hargest R, Martin M, Delicata RJ. Intra-abdominal hypertension and the abdominal compartment syndrome. Br J Surg 2004;91:1102-10.

3. Loftus IM, Thompson MM. The abdominal compartment syndrome following aortic surgery. Eur J Vasc Endovasc Surg 2003;25:97-109.

4. Meldrum DR, Moore FA, Moore EE, Franciose RJ, Sauaia A, Burch JM. Prospective characterization and selective management of the abdominal compartment syndrome. Am J Surg 1997;174:667-72, discussion 663-72.

5. Ertel W, Oberholzer A, Platz A, Stocker R, Trentz O. Incidence and clinical pattern of the abdominal compartment syndrome after "damage-control", laparotomy in 311 patients with severe abdominal and/or pelvic trauma. Crit Care Med 2000;28:1747-53.

6. Raeburn CD, Moore EE, Biffl WL, Johnson JL, Meldrum DR, Offner PJ, Franciose RJ, Burch JM. The abdominal compartment syndrome is a morbid complication of postinjury damage control surgery. Am J Surg 2001;182:542-6.

7. Obeid F, Saba A, Fath J, Guslits B, Chung R, Sorensen V, Buck J, Horst M. Increases in intra-abdominal pressure affect pulmonary compliance. Arch Surg 1995;130:544-7, (discussion 547-8).

8. Cullen DJ, Coyle JP, Teplick R, LongMC. Cardiovascular, pulmonary, and renal effects ofmassively increased intra-abdominal pressure in critically ill patients. Crit Care Med 1989;17:118-21. 
9. Malbrain ML. Different techniques to measure intra-abdominal pressure (IAP): time for a critical re-appraisal. Intensive Care Med 2004;30:357-71.

10. Kron IL, Harman PK, Nolan SP. The measurement of intraabdominal pressure as a criterion for abdominal re-exploration. Ann Surg 1984;199:28-30.

11. Iberti TJ, Kelly KM, Gentili DR, Hirsch S, Benjamin E. A simple technique to accurately determine intra-abdominal pressure. Crit Care Med 1987;15:1140-2.

12. Sugrue M. Intra-abdominal pressure: time for clinical practice guidelines?. Intensive Care Med 2002;28:389-91.

13. Balogh Z, Jones F, D’Amours S, Parr M, Sugrue M. Continuous intra-abdominal pressure measurement technique. Am J Surg 2004;188:679-84.

14. Bland JM, Altman DG. Comparing methods of measurement: why plotting difference against standard method is misleading. Lancet 1995;346:1085-7.

15. Bland JM, Altman DG. Statistical methods for assessing agreement between two methods of clinical measurement. Lancet 1986;1:307-10.

16. Malbrain ML, Deeren D, De Potter TJ. Intra-abdominal hypertension in the critically ill: it is time to pay attention. Curr Opin Crit Care 2005;11:156-71.

17. Balogh Z, McKinley BA, Cox CS Jr, Allen SJ, Cocanour CS, Kozar RA, Moore EE, Miller IC, Weisbrodt NW, Moore FA. Abdominal compartment syndrome: the cause or effect of postinjury multiple organ failure. Shock 2003;20:483-92.

18. Collee GG, Lomax DM, Ferguson C, Hanson GC. Bedside measurement of intraabdominal pressure (IAP) via an indwelling naso-gastric tube: clinical validation of the technique. Intensive Care Med 1993;19:478-80.

19. Sugrue M, Buist MD, Lee A, Sanchez DJ, Hillman KM. Intraabdominal pressure measurement using a modified nasogastric tube: description and validation of a new technique. Intensive Care Med 1994;20:588-90. 
20. Vantrappen G, Janssens J, Peeters TL. The migrating motor complex. Med Clin North Am 1981;65:1311-29.

21. Moore FA, Cocanour CS, McKinley BA, Kozar RA, DeSoignie RC, Von-Maszewski ME, Weisbrodt NW. Migrating motility complexes persist after severe traumatic shock in patients who tolerate enteral nutrition. J Trauma 2001;51:1075-82.

22. Schachtrupp A, Tons C, Fackeldey V, Hoer J, Reinges M, Schumpelick V. Evaluation of two novel methods for the direct and continuous measurement of the intra-abdominal pressure in a porcine model. Intensive Care Med 2003;29:1605-8.

23. Engum SA, Kogon B, Jensen E, Isch J, Balanoff C, Grosfeld JL. Gastric tonometry and direct intraabdominal pressure monitoring in abdominal compartment syndrome. $\mathrm{J}$ Pediatr Surg 2002;37:214-8.

24. Fusco MA, Martin RS, Chang MC. Estimation of intra-abdominal pressure by bladder pressure measurement: validity and methodology. J Trauma 2001;50:297-302.

25. Brooks AJ, Simpson A, Delbridge M, Beckingham IJ, Girling KJ. Validation of direct intraabdominal pressure measurement using a continuous indwelling compartment pressure monitor. J Trauma 2005;58:830-2.

26. Shafik A, El-Sharkawy A, Sharaf WM. Direct measurement of intra-abdominal pressure in various conditions. Eur J Surg 1997;163:883-7.

27. Cheatham ML, White MW, Sagraves SG, Johnson JL, Block EF. Abdominal perfusion pressure: a superior parameter in the assessment of intra-abdominal hypertension. $\mathbf{J}$ Trauma 2000;49:621-6, (discussion 626-7). 
Figure 1. Continuous direct intra-abdominal pressure set-up: a central venous catheter was placed through the abdominal wall in the anterior axillary line $\pm 5 \mathrm{~cm}$ cranial of the iliac crest, and connected to a pressure transducer.

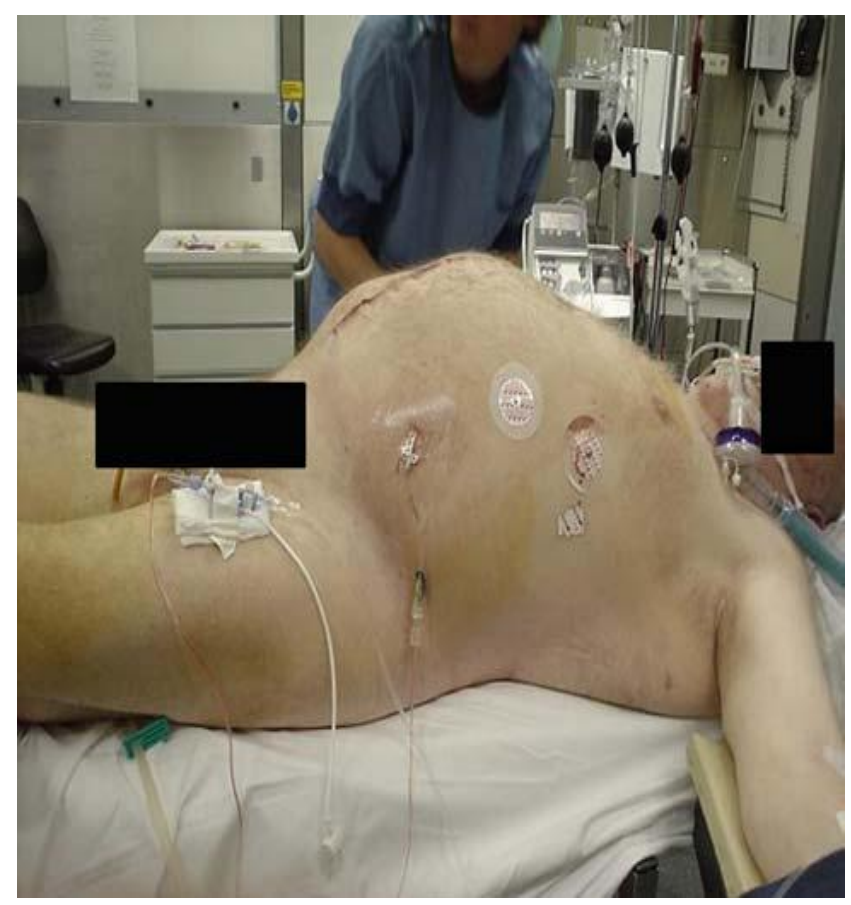

Figure 2. Set-up for measuring intra-abdominal pressure using the Kron method.

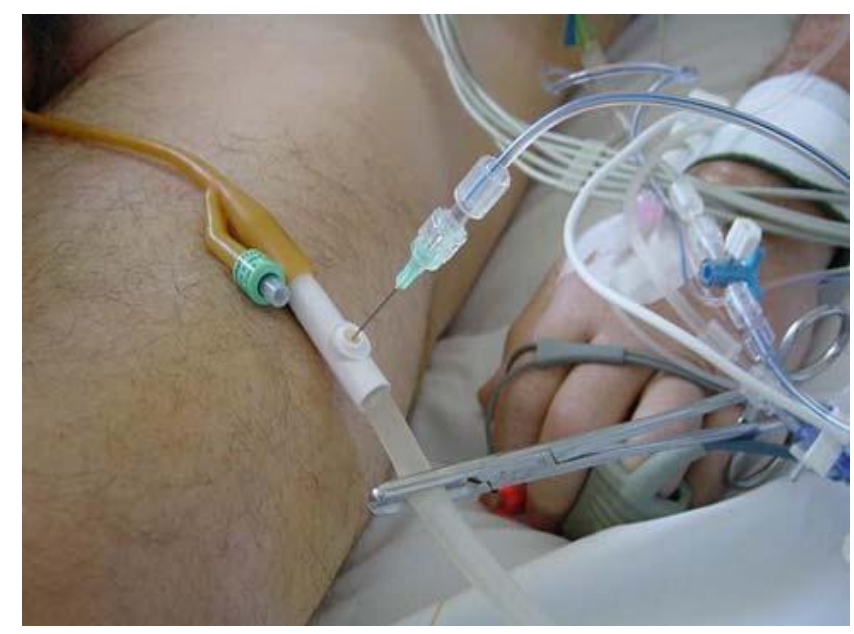


Figure 3. Bland-Altman plot: bladder pressure (BP) and continuous direct intra-abdominal pressure (CDIAP). Mean difference: -1.6 ; SD of differences: $2.7 \mathrm{mmHg}$.

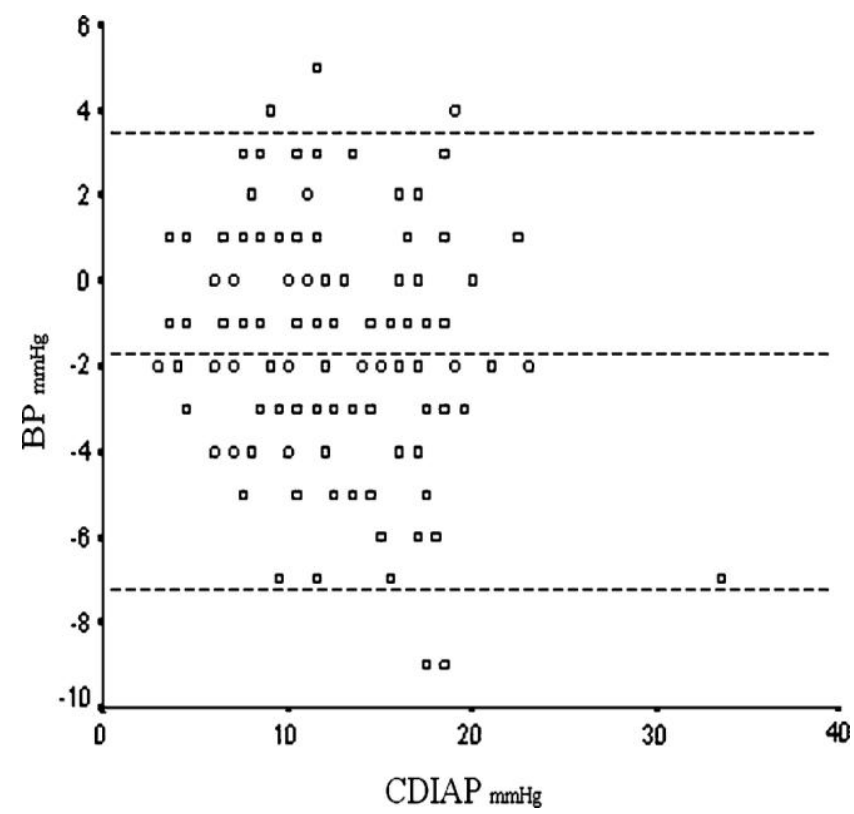

Figure 4. Bladder pressure (BP) versus continuous direct intraabdominal pressure (CDIAP). Intraclass correlation: 0.82 .

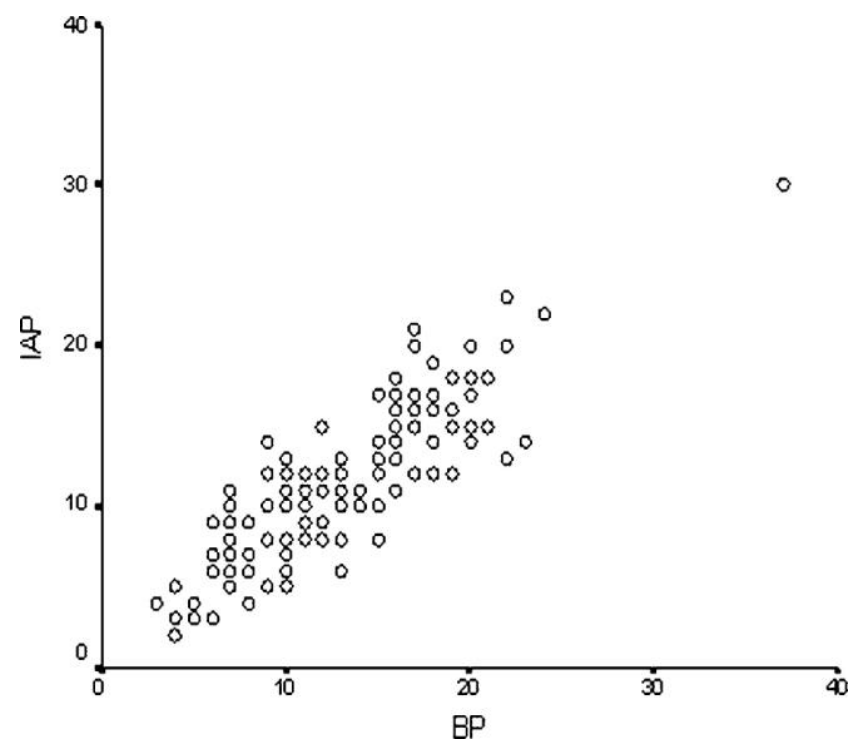

